\title{
Multiphase coexistence in polydisperse colloidal mixtures
}

\author{
C. Grodon and R. Roth \\ Max-Planck-Institut für Metallforschung, \\ Heisenbergstr. 3, D-70569 Stuttgart, Germany \\ and \\ Institut für Theoretische und Angewandte Physik, \\ Universität Stuttgart, Pfaffenwaldring 57, \\ D-70569 Stuttgart, Germany
}

\begin{abstract}
We study the phase behavior of mixtures of monodisperse colloidal spheres with a depletion agent which can have arbitrary shape and can possess a polydisperse size or shape distribution. In the low concentration limit, considered here, we can employ the free-volume theory and take the geometry of particles of the depletion agent into account within the framework of fundamental measure theory. We apply our approach to study the phase diagram of a mixture of (monodisperse) colloidal spheres and two polydisperse polymer components. By fine tuning the distribution of the polymer it is possible to construct a complex phase diagram which exhibits two stable critical points.
\end{abstract}

\section{INTRODUCTION}

In the study of the phase behavior of colloidal mixtures there are two very distinct approaches. From a theoretical point of view one would like to keep the description of the mixture as simple as possible in order to be able to calculate the phase diagram using well established theoretical tools. Furthermore, it is possible to understand the main physics, i.e. the phase behavior, bulk correlation functions, or inhomogeneous density distributions, in simple terms. One example of such an theoretical approach is the Asakura-Oosawa-Vrij (AOV) model for colloid-polymer mixtures $1,2,3$ Within the AOV model, colloids are treated as hard spheres, while polymer behave like hard spheres only when interacting with colloids. The polymer-polymer interaction vanishes. Based on this model, one finds that the phase diagram of a colloid-polymer mixture, assuming perfectly monodisperse colloids and polymer, resembles that of a simple fluid, and the reservoir polymer density plays the role of an inverse temperature. One, relatively simple but successful, theoretical approach for treating the phase behavior of this model colloid-polymer mixture is the freevolume theory (FVT) that assumes a low concentration of the depletion agent, the polymer $\stackrel{4}{*}$

A very different picture of the phase behavior of colloidal mixture originates from experimental studies. While in some cases it is possible to prepare model systems with almost monodisperse colloids and polymer, $, 5,6$ the generic case of a colloidal sample is polydisperse due to imperfections of the synthesis process. Polydispersity can be found both in size and in shape distributions. From the theoretical point of view, polydispersity is often considered as a defect, because it complicates calculations. However, polydispersity can give rise to interesting behavior, which can be utilized in experiments,,$\frac{7}{-}$ if the role of polydispersity is sufficiently well understood. One example is the study of the glass transition, where polydispersity in model hard colloidal spheres has the role as to suppress the freezing transition at high density of colloids so that a glass can form. $\frac{8}{8}$

There are various very different ways to achieve a successful description. For hard and permeable spheres Salacuse and Stell ${ }^{9}$ showed a thermodynamic approach based on the Percus-Yevick equation of state and the more accurate approach by Mansoori et al. The polydispersity in the chain-length of self-excluding polymer has been treated in Ref. 10. Polydispersity has been incorporated in the theory in different manner. Nearmonodisperse distributions have been treated in a perturbative fashion ${ }^{11}$ and bimodal size distributions has been studied in Ref. 12 .

In the present study we wish to increase the understanding of effects that can be caused by polydispersity further. To this end we consider a mixture of monodisperse colloidal spheres with a polydisperse depletion agent. We keep the theory simple by assuming that the depletion agent is efficient and can produce a sufficiently strong depletion attraction between colloidal spheres at rather low concentrations. In this limit we can generalize the free-volume theory within the framework of fundamental measure theory ${ }^{13}$ (FMT) to cases of polydisperse size or shape distributions.

As an application of our general and powerful approach we study the phase behavior of a ternary mixture of (monodisperse) colloidal spheres with two polydisperse polymer components with distinct size difference. Our study shows that the distribution of the depletion agent can be employed to tune the phase behavior of colloidal mixtures in a wide range and it is possible to generate a third fluid phase. Beside the low density (in colloids) gas and a high density liquid, we find a low density liquid phase. This is similar to findings in systems in which two length scales are introduced in the inter-particle interaction potentials $14,15,16$

The paper is structured as follows. In Section II we recall the theoretical basics of free-volume theory within the framework of FMT. We extend the approach of Ref. 17 to treat discrete and continuous distributions of the depletion agent. In Section III we discuss a few dis- 
tribution functions which are frequently used in studies of polydisperse systems and show that for infinitely thin needles or platelets various types of size distributions lead to explicit expressions for the effective free-volume frac-

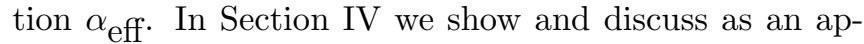
plication of our theory the phase diagrams for the case of a ternary mixture of (monodisperse) colloidal spheres and two polydisperse polymer components. We discuss the results and conclude in Section $\mathrm{V}$

\section{THEORY}

We study mixtures of colloidal spheres immersed in a solvent of depletion agent. The latter component can possess a polydisperse size or shape distribution. Here we are mainly interested in mixtures where the depletion potential between the spheres is strongly attractive already at rather low concentrations of the depletion agent. In this case we can employ the description of Asakura, Oosawa ${ }^{1.2}$ and $\mathrm{Vrij}^{\underline{3}}$ for the mutual particle interactions, which keeps the model simple. The colloid-colloid and the colloid-depletion agent interactions are modeled as hard-core interactions, while the mutual interaction between particles of the depletion agent vanish. It has been shown 18 that the phase behavior of the Asakura-OosawaVrij model is closely related to the free-volume theory, in which the excess free energy of the mixture is expanded up to first order in the density of the depletion agent. This linearization of the excess free energy is equivalent to a vanishing interaction among particles of the depletion agent. As $\mathrm{FMT}^{13}$ provides a good framework for the free-volume theory we first recall its main ideas as presented in Refs. 19 and 17 for discrete mixtures and generalize it to continuous distributions of the depletion agent.

\section{A. Free-volume theory}

In order to describe the phase behavior of colloidal mixtures one has first to choose the thermodynamic ensemble. For our purpose a system with a fixed number of big spherical colloidal particles $N_{c}$, which is coupled to a reservoir of a depletion agent at chemical potential $\mu_{d}$ or fugacity $z_{d}$, respectively, represents the best choice ${ }^{20}$ Hence, the system is treated in a semi-grand canonical ensemble. The Helmholtz free energy separates into two terms

$$
\begin{aligned}
\beta F\left(N_{c}, V, z_{d}\right)= & \beta F\left(N_{c}, V, z_{d}=0\right) \\
& +\int_{0}^{z_{d}} \mathrm{~d} z_{d}^{\prime}\left(\frac{\partial \beta F\left(N_{c}, V, z_{d}^{\prime}\right)}{\partial z_{d}^{\prime}}\right) .
\end{aligned}
$$

The first term is the Helmholtz free energy of pure colloids. By Taylor expansion of the integrand of the second term in Eq. (1) up to first order in $z_{d}$ we obtain an expression for the volume available to particles of the depletion agent in a system of $N_{c}$ colloids at low concentrations of the depletion agent, the so-called free volume denoted by $\alpha V$. In this limit of small fugacity of the depletion agent, we can rewrite Eq. (1) into

$$
\beta F\left(N_{c}, V, z_{d}\right)=\beta F_{0}\left(N_{c}, V\right)-\rho_{d}^{r}\left(z_{d}\right) \alpha V,
$$

where $\rho_{d}^{r}$ is the reservoir density of the depletion agent. Within the AOV model the free-volume fraction $\alpha$ depends only on the geometry of the depletion agent, e.g. the size ratio $q=\sigma_{d} / \sigma_{c}$ in the case of spherical polymer, and on the packing fraction of the colloidal spheres $\eta_{c}=\pi \sigma_{c}^{3} N_{c} /(6 V)$.

For the calculation of the phase diagram of the colloidal mixture we require the chemical potential of the colloid component $\mu_{c}$ and the osmotic pressure $p$. In the bulk two phases I and II coexist if they are in chemical and mechanical equilibrium, i.e. if $\mu_{c}^{\mathrm{I}}=\mu_{c}^{\mathrm{II}}$ and $p^{\mathrm{I}}=p^{\mathrm{II}}$, respectively. The chemical potential $\mu_{c}$ and the pressure $p$ follow from the Helmholtz free energy via 20

$$
\mu_{c}=\left.\frac{\partial F}{\partial N_{c}}\right|_{V, z_{d}}=\mu_{0}\left(\eta_{c}\right)-\rho_{d}^{r}\left(z_{d}\right)\left(\frac{\partial \alpha}{\partial \eta_{c}}\right) \frac{\pi \sigma_{c}^{3}}{6}
$$

and

$$
p=\left.\frac{\partial F}{\partial V}\right|_{N_{c}, z_{d}}=p_{0}\left(\eta_{c}\right)+\rho_{d}^{r}\left(z_{d}\right)\left(\alpha-\eta_{c} \frac{\partial \alpha}{\partial \eta_{c}}\right) .
$$

The chemical potential and the pressure of the pure reference system, labeled $\mu_{0}$ and $p_{0}$, are described accurately by the Carnahan-Starling expressions in the fluid phase. To assure thermodynamic consistency the free-volume fraction $\alpha$ must also be calculated from the equation of state of the reference system, i.e. the Carnahan-Starling equation of state $\stackrel{17}{ }$ Besides coexisting fluid states, i.e. points on the binodal line in phase diagram, we calculate the spinodal line which separates metastable states from thermodynamically unstable ones. For thermodynamically unstable states the second derivative of the Helmholtz free energy w.r.t. the colloid packing fraction $\eta_{c}$ is negative and for points on the spinodal line it vanishes, i.e. $\partial^{2} F / \partial \eta_{c}^{2}=0$.

For the solid-fluid coexistence we need the chemical potential and equation of state of a reference (fcc) hardsphere crystal, which is described by 21

$$
\beta \mu=2.1306+3 \ln \left(\frac{\eta_{c} \eta_{c p}}{\eta_{c p}-\eta_{c}}\right)+\frac{3 \eta_{c p}}{\eta_{c p}-\eta_{c}}
$$

with $\eta_{c p}=\pi \sqrt{2} / 6 \approx 0.74$, the packing fraction for close packing, and ${ }^{22}$

$$
\beta p v_{s}=\frac{3 \eta_{c} \eta_{c p}}{\eta_{c p}-\eta_{c}}
$$

\section{B. Fundamental measure theory}

For discrete mixtures of two species the excess Helmholtz free energy density $\Phi=\beta F / V$ is constructed 
from two sets of weighted densities $\left\{n_{i}^{c}\right\}$ and $\left\{n_{i}^{d}\right\}$, one for the colloids $c$ and one for the depletion agent $d \underline{18}$ In homogeneous bulk fluids $i$ labels four scalar-weighted densities, which can be identified as the scaled-particle theory variables $\zeta_{i}$ of the $N$-component mixture ${ }^{23,24}$

$$
n_{i} \equiv \zeta_{i}=\sum_{\nu=1}^{N} g_{\nu}^{(i)} \rho_{\nu}
$$

with the geometrical measures of component $\nu$ : volume $g_{\nu}^{(3)} \equiv v_{\nu}$, surface area $g_{\nu}^{(2)} \equiv a_{\nu}$, integrated mean curvature $g_{\nu}^{(1)} \equiv c_{\nu}$ and Euler characteristics $g_{\nu}^{(0)} \equiv X_{\nu}$.

An explicit expression for the free energy density of the AOV mixture in the limit of low density of the depletion agent can be obtained by expanding the hard-sphere (HS) mixture free energy with weighted densities $n_{i}=n_{i}^{c}+n_{i}^{d}$ up to linear order in the density $\rho_{d}: 17,18$

$$
\Phi^{\mathrm{AOV}}\left(\left\{n_{i}^{c}\right\},\left\{n_{i}^{d}\right\}\right)=\Phi^{\mathrm{HS}}\left(\left\{n_{i}^{c}\right\}\right)+\sum_{k=0}^{3} \frac{\partial \Phi\left(\left\{n_{i}^{c}\right\}\right)}{\partial n_{k}^{c}} n_{k}^{d}(8)
$$

In order to relate the terms in Eq. (8) to the free-volume fraction $\alpha$ we transform the excess Helmholtz free energy density, which in Eq. (11) is given in the semi-grand canonical ensemble, into the canonical ensemble and obtain in terms of the colloid packing fraction $\eta_{c}$ and the density $\rho_{d}$

$$
\Phi\left(\eta_{c}, \rho_{d} ; q\right)=\Phi\left(\eta_{c}\right)-\rho_{d} \ln \left(\alpha\left(\eta_{c} ; q\right)\right) .
$$

By direct comparison of Eq. (8) and Eq. (9) we obtain a FMT expression for the free-volume fraction ${ }^{17}$

$$
\alpha\left(\eta_{c} ;\left\{g_{\nu}^{(i)}\right\}\right)=\exp \left(-\sum_{k=0}^{3} \frac{\partial \Phi\left(\left\{n_{i}^{c}\right\}\right)}{\partial n_{k}^{c}} g_{d}^{(k)}\right) .
$$

The partial derivatives of the free energy w.r.t. the weighted densities are thermodynamic quantities of the (pure) colloids, namely, the pressure $p_{0}$, the surface tension $\gamma$ at a planar hard wall and the bending rigidities $\kappa$ and $\bar{\kappa}$, which describe the effect on the free energy due to curved surfaces $\frac{17,25,26}{1}$ The depletion agent enters the expression for the free-volume fraction $\alpha$ only through its geometrical measures $g_{d}^{(k)}$. For the free energy density we employ Rosenfeld's original formulation of $\mathrm{FMT}^{13}$ and the more accurate White Bear version from Refs, $\underline{27,28}$

$$
\begin{aligned}
\Phi\left(\left\{n_{\alpha}\right\}\right)= & -n_{0} \ln \left(1-n_{3}\right)+\frac{n_{1} n_{2}}{1-n_{3}} \\
& +\frac{n_{2}^{3}\left(n_{3}+\left(1-n_{3}\right)^{2} \ln \left(1-n_{3}\right)\right)}{36 \pi n_{3}^{2}\left(1-n_{3}\right)^{2}},
\end{aligned}
$$

which is based on the Boublik-Mansoori-CarnahanStarling-Leland (BMCSL) equation of state 29,30

$$
\begin{aligned}
\beta p_{B M C S L}= & \frac{n_{0}}{1-n_{3}}+\frac{n_{1} n_{2}}{\left(1-n_{3}\right)^{2}}+\frac{n_{2}^{3}}{12 \pi\left(1-n_{3}\right)^{3}} \\
& -\frac{n_{3} n_{2}}{36 \pi\left(1-n_{3}\right)^{3}} .
\end{aligned}
$$

It would also be possible to base the free-volume theory on a recent, slightly more consistent, mixture equation of state. ${ }^{26,31}$

\section{Generalization to polydisperse depletion agent}

FMT is a mixture theory by construction. For mixtures of colloids and ideal depletion agent we can employ parameters $q_{i}$ to parameterize the geometry of particles of the depletion agent components. Although it is straightforward within FMT to treat general mixtures of $\nu_{c}$ colloid and $\nu_{d}$ depletion agent components, we will in the following restrict our studies to the simpler case of a single colloid species with packing fraction $\eta_{c}$. For the depletion agent we start by considering $\nu_{d}$ components. In the semi-grand canonical ensemble the excess Helmholtz free energy density is, analogous to Eq. (9), given by

$$
\Phi\left(\eta_{c},\left\{\eta_{d, i}^{r}\right\} ;\left\{q_{i}\right\}\right)=\Phi_{c}-\sum_{\nu=1}^{\nu_{d}} \rho_{d, i}^{r} \alpha\left(\eta_{c} ;\left\{q_{i}\right\}\right),
$$

and the set $\left\{g_{\nu}^{(i)}\right\}$ of geometrical measures is characterized by the set of parameters $\left\{q_{i}\right\}$. To study the influence of polydispersity in the depletion agent on the phase behavior it might be sufficient to consider a discrete mixture, however we prefer to introduce continuous distributions, which can represent experimental systems more accurately than a discrete distribution. In the following we restrict the set $\left\{g_{\nu}^{(i)}\right\}$ to depend on a single parameter $q$ only. We introduce $d(q)$ as a continuous distribution of the depletion agent in the reservoir, and require that $\int d(q) \mathrm{d} q=1$. The density distribution of the depletion agent follows directly: $\rho_{d}^{r}(q)=\rho_{d}^{r} d(q)$. By specifying the fundamental geometrical measures of the depletion agent as function of $q$ we obtain, similar to Ref. 32 ,

$$
\begin{aligned}
& \Phi^{p d}\left(\eta_{c}, \rho_{d}^{r} ;[d]\right)=\Phi\left(\eta_{c}\right) \\
& -\rho_{d}^{r} \int \mathrm{d} q d(q) \alpha\left(\eta_{c}, v_{d}(q), a_{d}(q), c_{d}(q), X_{d}(q)\right),
\end{aligned}
$$

which is a functional of the distribution $d(q)$. Note that the precise meaning of $q$ is unspecified so far and can refer to a size ratio, in the case of spherical mixtures, or to a parameter that specifies the shape of the depletion agent in a more complicated manner. We return to this point later. Analogous to Eq. (2) we call the integral on the r.h.s. of Eq. (14) the effective free-volume fraction $\alpha_{\text {eff }}$ and rewrite Eq. (14) as

$$
\Phi^{p d}\left(\eta_{c}, \rho_{d}^{r} ;[d]\right)=\Phi\left(\eta_{c}\right)-\rho_{d}^{r} \alpha_{\mathrm{eff}}\left(\eta_{c} ;[d]\right) .
$$

\section{DISTRIBUTION FUNCTIONS}

To study the phase behavior of a mixture of spherical colloids and a polydisperse depletion agent we focus on three frequently used distributions, namely the Schulz 
$(S)$, Gaussian $(G)$ and the Hat-like $(H)$ distributions. These distributions are characterized by two parameters corresponding to its first and second moment. The average asymmetry ratio is given by $\bar{q}=\tilde{\sigma}_{d} / \sigma_{c}$, with the colloid diameter $\sigma_{c}$ and a length-scale of the depletion agent $\tilde{\sigma}_{d}$. The parameter $z$ describes the degree of polydispersity: the limiting case $z \rightarrow \infty$ equals the monodisperse fluid and by decreasing the value of $z$ the distribution is broadened. The distributions under consideration here lead to explicit expressions for the effective free-volume fraction $\alpha_{\text {eff }}\left(\eta_{c} ; d(q ; \bar{q}, z)\right)$, if the geometries of the depletion agent is simple. For more complicated shapes we have to perform the integration numerically. In terms of $\bar{q}$ and $z$ the distributions considered here are given by

$$
\begin{aligned}
d_{S}(q ; \bar{q}, z)= & \left(\frac{z}{\bar{q}}\right)^{z} q^{z-1} \frac{\exp (-z q / \bar{q})}{\Gamma(z)}, \quad z \geq 1, \\
d_{G}(q ; \bar{q}, z)= & \frac{z}{\sqrt{\pi}} \exp \left(-(q-\bar{q})^{2} z^{2}\right) \\
& \times 2 \Theta(q) /[\operatorname{erf}(z \bar{q})+1], \\
d_{H}(q ; \bar{q}, z)= & \frac{z}{2} \Theta\left(q-\bar{q}+z^{-1}\right) \\
& \times \Theta\left(-q+\bar{q}+z^{-1}\right), \quad \bar{q} z>1 .
\end{aligned}
$$

It is evident that $q>0$ is required in all distributions and the integration in $\alpha_{\text {eff }}\left(\eta_{c} ; d(q ; ; \bar{q}, z)\right)$ is performed in the limits from 0 to $\infty$. In the case of a Gaussian distribution one can obtain simpler explicit expressions by imposing the full integration range $q=-\infty$ to $\infty$. The result, $d_{G}^{\prime}$, is similar to that of the cut Gaussian distribution $d_{G}$

$$
d_{G}^{\prime}(q ; \bar{q}, z)=\frac{z}{\sqrt{\pi}} \exp \left(-(q-\bar{q})^{2} z^{2}\right) .
$$

Obviously, it is possible to consider different size distributions such as the log-normal distribution, which decays slower for large values of $q$ than the distributions considered here.

\section{A. Explicit expressions for $\alpha_{\text {eff }}$}

The equations for the chemical potential $\mu_{c}$ and the osmotic pressure $p$ from Eqs. (3) and (4) for polydisperse size distributions contain integrals over the distribution. For the calculation of the phase diagram these equations must be solved simultaneously. In general this must be done numerically. In the case of one- and two-dimensional depletion agents, such as infinitely thin rods or platelets, their geometry is sufficiently simple so that the effective free-volume fraction $\alpha_{\text {eff }}$ can be calculated explicitly. Infinitely thin needles represent an onedimensional depletion agent. Infinitely thin platelets represent a two-dimensional depletion agent. Here we consider both disk- and hexagon-shaped platelets. The geometric measures for these geometries are given in Tab. [1. In Appendix $\mathrm{A}$ we show explicit expressions for $\alpha_{\mathrm{eff}}$ for one- and two-dimensional depletion agents and the distributions given above. As aforementioned, we obtain

\begin{tabular}{cl|l|cccc}
\hline Dim & Depletion agent & Geometry & $v_{d}$ & $a_{d}$ & $c_{d}$ & $X_{d}$ \\
\hline \hline 1 & needle & $L=q \sigma_{c}, R=0$ & 0 & 0 & $\frac{L}{4}$ & 1 \\
\hline 2 & disk & $R=q \sigma_{c} / 2, h=0$ & 0 & $2 \pi R^{2}$ & $\frac{\pi}{4} R$ & 1 \\
2 & hexagon & $R=q \sigma_{c} / 2, h=0$ & 0 & $3 \sqrt{3} R^{2}$ & $\frac{3}{4} R$ & 1 \\
\hline
\end{tabular}

TABLE I: Geometrical measures for one- and two-dimensional depletion agents. All lengths are measured in units of the diameter $\sigma_{c}$ of the spherical colloids. We consider needles of length $L$ and radius $R=0$ and platelets with radius $R$ and thickness $h=0$. The value of $q$ plays the role of a size ratio and compares the size of the depletion agent to that of a colloid.

the thermodynamic quantities of the pure hard-sphere fluid that enter the free-volume fraction $\alpha_{\text {eff }}$ from the excess free energy density $\Phi$ of the White Bear version of FMT $\stackrel{27,28}{2}$ These quantities are the pressure $\beta p=$ $\partial \Phi / \partial n_{3}$, the surface tension $\beta \gamma=\partial \Phi / \partial n_{2}$ and bending rigidities $\beta \kappa=\partial \Phi / \partial n_{1}$ and $\beta \bar{\kappa}=\partial \Phi / \partial n_{0}, \frac{17,25,26}{1}$

In the present study we restrict the product $\bar{q} z$ to sufficiently large values. For the Hat-like distribution we require $\bar{q} z>1$ which ensures that $\int_{0}^{\infty} \mathrm{d} q d(q)=1$. In the case of the Gaussian distribution $d_{G}^{\prime}$ we require $\bar{q} z>1$ because for small values of $\bar{q} z$ the contribution of the distribution from values $q<0$ increases, e.g. for $\bar{q}=0.5$ and $z=3$ we obtain $\int_{-\infty}^{0} \mathrm{~d} q d_{G}(q) \sim \mathcal{O}\left(10^{-2}\right)$.

In the case of two-dimensional depletion agents, the geometric measures can differ for different geometry of the depletion agent: for disk- and hexagonal platelets $a_{d}$ and $c_{d}$ differ - see Tab. II. For that reason we show the result for $\alpha_{\text {eff }}$ in Appendix $\mathrm{A}$ in its general form.

\section{RESULTS}

While our approach developed in Section III is general and allows one to study various systems, we want to present only one application. We study the phase behavior of a ternary mixture of (monodisperse) colloidal spheres with two polydisperse polymer components of distinct sizes. Both polymer components possess a size distribution which we describe by Schulz distributions, which in total lead to $d_{S}\left(q ; \bar{q}, \bar{Q}, z_{\bar{q}}, z_{\bar{Q}}\right)=$ $x d_{S}\left(q ; \bar{q}, z_{\bar{q}}\right)+(1-x) d_{S}\left(q ; \bar{Q}, z_{\bar{Q}}\right)$ with a mixing parameter $x$ and two averaged size ratios $\bar{q}$ and $\bar{Q}$. For simplicity we assume the width of both parts of the distribution to be equal. The polymer reservoir packing fraction is given by $\tilde{\eta}_{d}^{r}=\frac{\pi}{6}\langle q\rangle^{3} \sigma_{c}^{3} \rho_{d}^{r}$, where $\langle q\rangle=x \bar{q}+(1-x) \bar{Q}$.

We expect the phase behavior to be most interesting if the average size ratios $\bar{q}$ and $\bar{Q}$ are distinct, so that the phase diagrams in the limiting cases $x=0$ and $x=1$ are sufficiently different. To this end we choose $\bar{q}=0.25$ and $\bar{Q}=2.0$. In the case of $x=0$ when we obtain a binary mixture with an averaged size ratio $\bar{Q}=2.0$, the phase diagram exhibits a stable fluid-fluid phase separation into a colloid poor gas and a colloid rich liquid phase. For the mixing parameter $x=1$, when the averaged size ratio $\bar{q}=0.25$, the fluid-fluid phase separa- 
tion, which is metastable w.r.t. to crystallization in the monodisperse case $\frac{19}{1}$ can be stabilized by a sufficient degree of polydispersity $33,34,35$

Before we study the full phase diagram of the ternary mixture, we determine which value of $z_{\bar{q}}$ is required in order to stabilize the fluid-fluid phase separation for $x=$ 1.

\section{A. Influence of size polydispersity}

In this section we study the phase diagram of a binary colloid-polymer mixture, which corresponds to the limiting case $x=1$, with an averaged size ratio of $\bar{q}=0.25$. In contrast to the aforementioned examples of one- and two-dimensional depletion agents, $\alpha_{\text {eff }}$ can not be obtained explicitly. Therefore, we have to determine the phase coexistence by solving two equations, Eqs. (3) and (41), numerically.

The equation of state of the pure reference system and the free-volume fraction $\alpha_{\text {eff }}$ respectively, and hence the chemical potential $\mu_{c}$ and the osmotic pressure $p$ of the colloids, are based on the free energy density $\Phi$ of the White Bear version of FMT, Eq. (11).

In Fig. 1(a) we show the phase diagrams of polydisperse AOV mixtures as we vary the degree of polydispersity. For the size ratio $\bar{q}=0.25$ and a narrow distribution of the polymer, the fluid-fluid phase separation remains metastable similar to the monodisperse case. We show the phase diagram for $z=50$ (full lines) in Fig. 1(a) and the corresponding size distribution in Fig. 1(b). Upon decreasing $z$, which broadens the size distribution, the fluidfluid and the fluid-solid coexistence lines come closer to each other and almost touch for $z=10$ (not shown). By further increasing the degree of polydispersity the fluidfluid coexistence becomes stable, as we show in Fig. 1(a) for $z=5$ (dashed-dotted lines) and $z=2$ (dotted lines). The corresponding size distributions are shown in Fig. 1(b). The critical point is shifted to lower colloid packing fractions $\eta_{c}$, as the value of $z$ decreases.

The polymer distribution in the system differs from that in the reservoir, 34 which is a phenomenon known as fractionation. For spherical polymer we find results equivalent to those of Ref. 34. At low packing fractions of the colloids the size distribution of (spherical) polymer follows closely the reservoir distribution. As the colloid packing fraction increases, the maximum of the polymer size distribution decreases significantly and moves towards smaller polymer radii. In principle we observe a qualitatively similar but less pronounced behavior also in the case of platelet-like or rod-like depletion agents. The maximum in the size distribution of the depletion agent becomes smaller and moves to smaller sizes as the colloid packing fraction in the system increases. This is to be expected, because one- or two-dimensional depletion agents require less free volume to fit between colloids than spherical polymer.

We have confirmed that a treatment of the phase be-
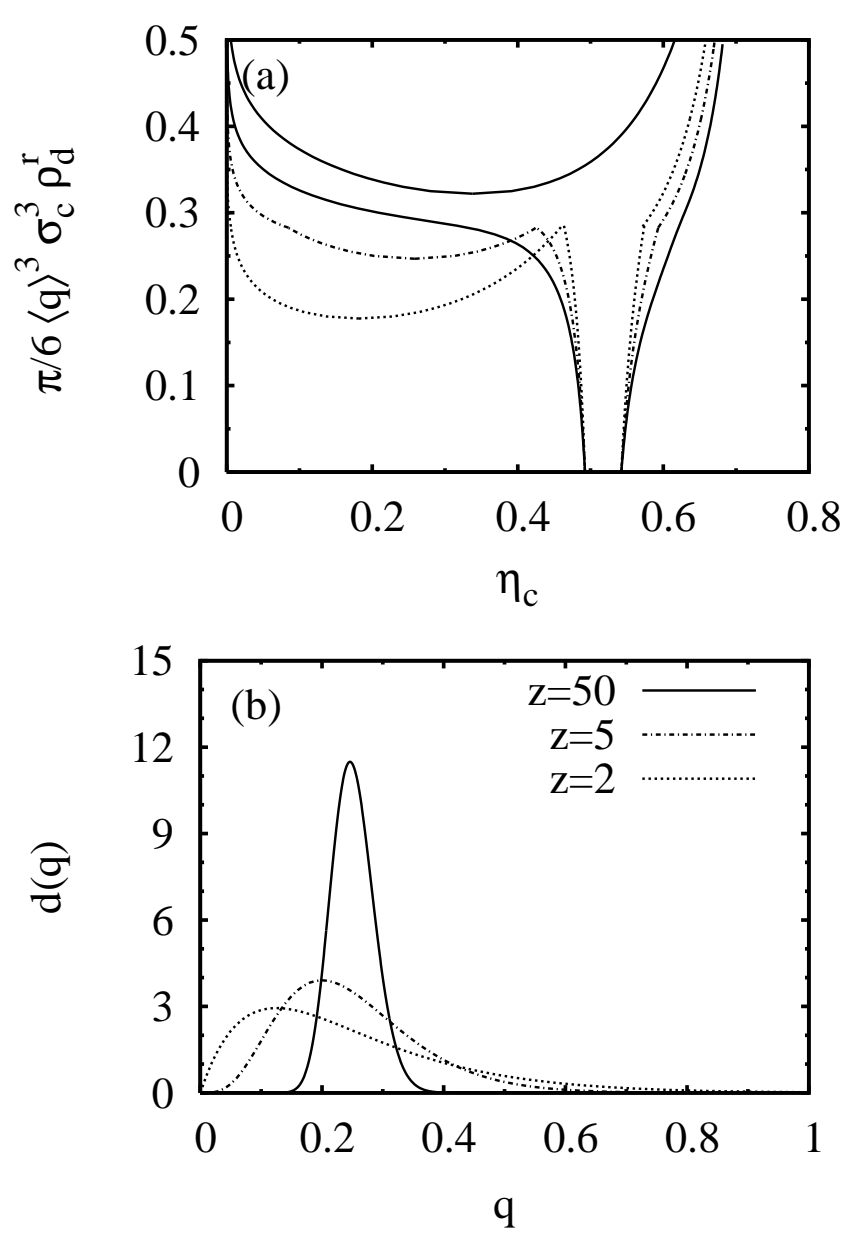

FIG. 1: (a) Phase diagrams of mixtures of colloids and polydisperse (spherical) polymer with an average size ratio $\bar{q}=0.25$ and (b) the corresponding distributions. We employ a free-volume theory based on the BMCSL equation of state. For low degrees of polydispersity, corresponding to $z=50$ (full lines), the fluid-fluid phase separation is metastable w.r.t. fluid-solid coexistence. Upon increasing the degree of polydispersity, corresponding to $z=5$ (dasheddotted lines) and $z=2$ (dotted lines), the fluid-fluid coexistence is stabilized.

havior completely based on the PY compressibility equation of state yield qualitatively similar results. In the present approach this is achieved by employing the free energy density $\Phi$ of Rosenfeld's original formulation of FMT to evaluate $\alpha_{\text {eff }}$ and the pressure and chemical potential for the pure reference system. The results in Refs. 33, 34, and 35 are somewhat in between these two predictions as their approach employs a hard-sphere reference system based on the Carnahan-Starling equation of state and a free-volume fraction based on the PY compressibility equation of state, which for spheres is equivalent to the scaled-particle theory expression. 


\section{B. Influence of shape polydispersity}

In this section we briefly address the question whether polydispersity, which describes a shape distribution, can also stabilize the fluid-fluid phase separation. One can think of the shape polydispersity in two different ways. On the one hand it can be thought as describing effects due to imperfections in the synthesis of colloidal particles. On the other hand, and this is more relevant in the present context, it can be seen as a simple model for the effect that polymer coils are not rigid and can change their average shape when squeezed in between colloids.

To this end we consider ellipsoids, with half-axes $a=b=\sigma_{d} /(2 \sqrt{q})$ and $c=q \sigma_{d} / 2$, for which the geometrical measures can be obtained explicitly. $\frac{17}{q}$ This case is relatively simple as a single parameter $q$ characterizes the shape while $\sigma_{d}=0.25 \sigma_{c}$ is fixed. Obviously, more complicated scenarios are possible. Note that for this choice of half-axes the volume $v_{d}=\pi \sigma_{d}^{3} / 6$ is kept fixed, while the surface area $a_{d}=\pi \sigma_{d}^{2}(1 / q+$ $\left.q^{3 / 2}\left(\operatorname{arccosh} 1 / q^{3 / 2}\right) / \sqrt{1 / q-q^{2}}\right) / 2$ and integrated mean curvature $c_{d}=\sigma_{d}\left(q+\left(\arccos q^{3 / 2}\right) / \sqrt{q-q^{4}}\right) / 4$ depend on $q$. The Euler characteristic $X_{d}=1$, independent of $q$. Here, the parameter $q$ describes the degree of deviation of the shape from a sphere: for small values of $q$ we obtain lens-shaped particles (oblates), while for large values of $q$ the particles become cigar-shaped (prolates). Similarly to the case of size polydispersity, we observe that an increasing degree of shape polydispersity stabilizes the fluid-fluid phase separation w.r.t. fluid-solid decomposition. In Fig. 2 we show an example for a narrow shape distribution with $z=50$ (full line), in which case the fluid-fluid critical point is metastable, and one for a broad distribution with $z=2$ (dotted line). For $z=2$ we find a stable fluid-fluid phase separation.

It seems that polydispersity in general favors a stable fluid-fluid phase separation because the polydisperse depletion agent can fill the free volume of the system more effectively than a monodisperse one. Even if the bigger particles of the distribution cannot find free volume in the system, the smaller ones still do. In terms of the depletion potential this would result in a longer range of the effective attraction between colloids,$\frac{36}{2}$ giving rise to a more negative effective second virial coefficient $B_{2}^{\text {eff }}$ which increases the tendency for fluid-fluid phase separation. 37 This effect seems to be robust against details of introducing polydispersity and can also be observed for a depletion agent with $a=b=\sigma_{d} / 2$ and $c=q \sigma_{d} / 2$, where all geometrical measures, except of the Euler characteristics, depend on $q$.

\section{Ternary mixtures with a bimodal polydisperse distribution}

Based on our findings in Section IVA we choose $z_{\bar{q}}=$ $z_{\bar{Q}}=5$ for the bimodal polydisperse polymer distribu-

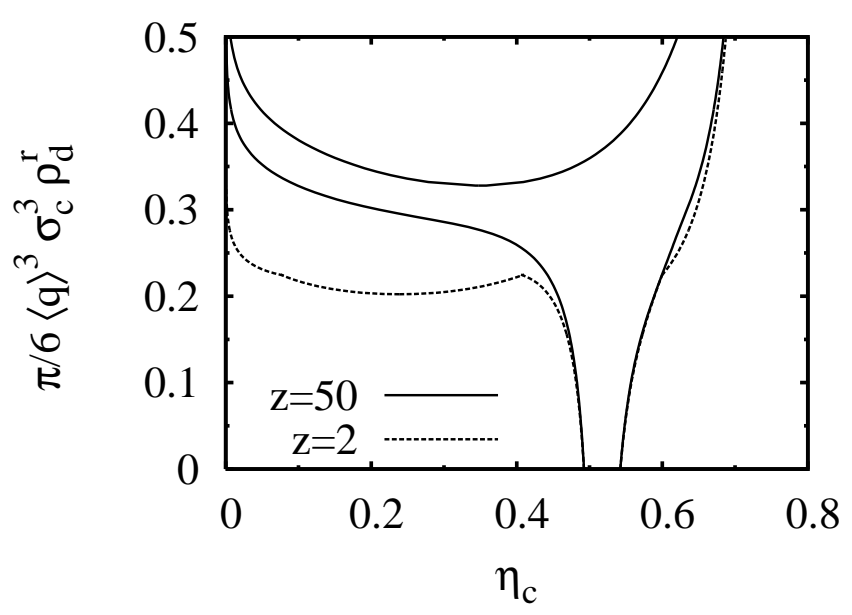

FIG. 2: Effect of shape polydispersity of the depletion agent on the phase behavior of a mixture of colloids and depletion agent. The depletion agent consists of ellipsoids with halfaxes $a=b=\sigma_{d} /(2 \sqrt{q})$ and $c=q \sigma_{d} / 2$. The mean value of the distribution is fixed at $\bar{q}=1.0$ while the size ratio between the depletion agent and the colloid is fixed at $\sigma_{d} / \sigma_{c}=0.25$. For $z=50$ (full lines) the fluid-fluid coexistence is metastable and similar to the monodisperse case (not shown here). Upon increasing the degree of polydispersity we find two stable fluid phases. The phase diagram for $z=2$ (dashed lines) exhibits a stable critical point and a triple point.

tion.

Before we show the calculated phase diagrams, it is interesting to compare the two stable critical points corresponding to the limiting cases, i.e. $x=1$ and $x=0$. The critical points can be found at very distinct regions of the phase diagram. In Fig. 3 we show the paths of the critical points through the phase diagram as a function of the mixing parameter $x$. When we start at $x=1$, for which only the smaller polymer are present, we find a critical point at $\eta_{c}=0.258$ and $\eta_{d}^{r}=0.247$. As we decrease the value of $x$, the critical point moves towards smaller values of $\eta_{c}$ until this critical point becomes metastable. For $x \lesssim 0.979$ the critical point no longer exists. When we start at $x=0$, for which only the larger polymer are present, we find a second critical point at $\eta_{c}=0.014$ and $\eta_{d}^{r}=0.924$. As the value of $x$ is increased we find that this critical point moves towards larger values of $\eta_{c}$ until it becomes metastable. For $x \gtrsim 0.997$ this critical point vanishes.

It is most interesting to note that both critical points found in this system, which correspond to critical points of the limiting cases, can exist at the same time for a range of values of the mixing parameter, $x \in[0.979,0.997]$. In order to obtain a full picture and determine where these critical points are stable we calculate the full phase diagrams in this regime.

In Figs. 4(a)-(b) we show phase diagrams in the $\eta_{c^{-}}$ $\tilde{\eta}_{d}^{r}$-representation for different values of $x$. For $x=$ 0.9999 [see Fig. 4(a)] the phase diagram is similar to the monodisperse case studied above. Upon decreasing $x$, the 
fraction of polymer with average size ratio $\bar{Q}$ increases, and the spinodal displays two minima, one corresponding to the stable critical point (triangle) and the second minimum corresponding to an additional metastable fluid-fluid phase separation (diamond) - see Fig. 4(b). Further decreasing the value of the mixing parameter to $x=0.9935$ [Fig. 4(c)] yield a phase diagram with two stable critical points and a second triple point. At one triple point, a gas, a liquid, and a solid phase coexist, while at the second triple point a gas phase and two liquid phases with different densities coexist. There is a novel low density liquid phase in the region between the two critical points with colloid packing fractions $\eta_{c}=0.0177$ and 0.251. As we vary the value of $x$ the triple points move. According to Gibbs' phase rule a four-phase coexistence between a gas, the low density liquid, the high density liquid and the solid is also possible. For our system it would be expected for $x$ between the values used in Fig. 4(b) and (c). In Fig. 4(d) for $x=0.98$ we also observe two critical points. The critical point with lower colloid packing fraction (diamond) is stable. The second critical point at higher colloid packing fraction (triangle) is now in the metastable regime and vanishes upon decreasing the values of $x$. For these systems one liquid phase and one triple point are stable. Further decreasing the values of $x$ below approximately 0.8 lead to a shift of the coexistence region to higher values of $\tilde{\eta}_{d}^{r}$. For $x \ll 1$ the fluid-fluid binodal is in the region of the fluid with monomodal polydisperse depletion agent with $\bar{Q}=2.0$.

We observe similar behavior for a mixture of colloids and two monodisperse polymer components, i.e. $z_{q}$ and $z_{Q} \rightarrow \infty$. For the same size asymmetries as above, $\bar{q} \rightarrow q=0.25$ and $\bar{Q} \rightarrow Q=2.0$, the critical point is metastable when $x=1$, or equivalently $\langle q\rangle=0.25$. We observe one stable and one metastable critical point for low colloid packing fraction upon decreasing the value of $x$. In the case $q=0.5$ and $Q=2.0$ there is a stable critical point for $x=1$. Decreasing $x$, similarly to the polydisperse case described above, leads to a second stable critical point. To summarize, we find that both by means of choosing the degree of polydispersity and the size asymmetry we can treat a model fluid which exhibits one or two (stable) critical points and one or two (stable) liquid phases. Furthermore, we have verified that it is possible to generate two critical points and hence three fluid phases by using a depletion agent of different than spherical shape.

\section{DISCUSSION}

We have generalized a novel approach to free-volume theory $\stackrel{17}{=}$ based on $\mathrm{FMT}^{13,23,24}$ to treat a depletion agent of arbitrarily shaped particles with a continuous distribution. Here, we introduced one parameter, $q$, to specify a continuously varying distribution of depletion agent.

To evaluate the phase diagram within FVT an expression for the free-volume fraction is required. In the case of

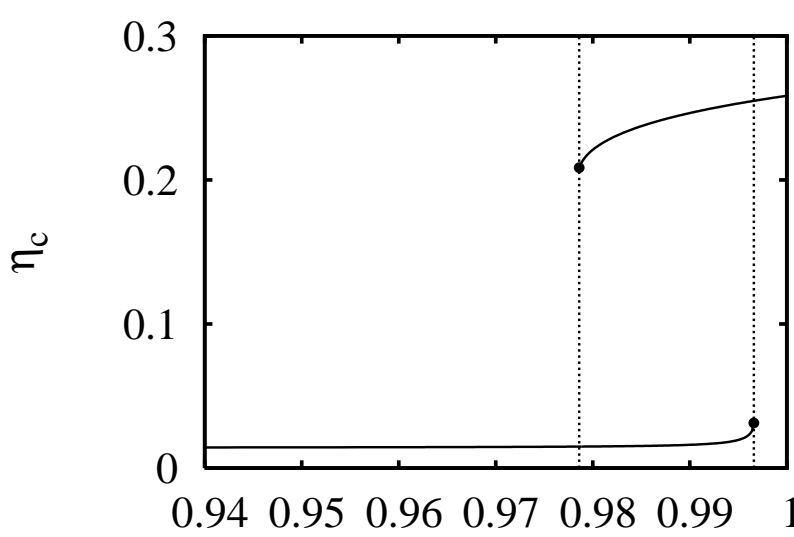

$\mathrm{X}$

FIG. 3: The path of the two critical points in a ternary mixture of colloidal spheres and two polydisperse polymer components. If the mixing parameter $x=1$, only the smaller polymer are present and we find a stable critical point at $\eta_{c}=0.258$ and $\eta_{d}^{r}=0.247$. As we decrease the value of $x$ this critical point moves towards smaller values of $\eta_{c}$ and vanishes at $x \approx 0.979$ (upper line). For a mixing parameter $x=0$, when only the bigger polymer are present, we find a second critical point at $\eta_{c}=0.014$ and $\eta_{p}^{r}=0.924$, which moves towards larger values of $\eta_{c}$ (lower line). For $x \in[0.979,0.997]$ (indicated by the dotted lines) we find two critical points in the system.

polydisperse size or shape distributions for the depletion agent, one can obtain an effective free-volume fraction, which in general has to be calculated numerically. In the cases of infinitely thin needles or platelets it is possible to get explicit expressions for the effective free-volume fraction for various distributions which are of interest from an experimental and a theoretical point of view. We give

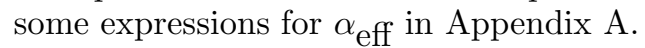

Beside size polydispersity, we have also considered shape polydispersity. To this end we have calculated phase diagrams of a mixture of colloidal spheres with penetrable ellipsoids with half-axes $a=b=\sigma_{d} /(2 \sqrt{q})$ and $c=q \sigma_{d} / 2$.

Our results confirm that polydispersity has the tendency to stabilize the fluid-fluid coexistence region w.r.t. crystallization. We have discussed this trend, in agreement with earlier studies $, 33,34,35$ in Section IVA for size polydisperse spherical polymer coils. In SectionIVB we have shown that similar behavior can be found for the case of shape polydisperse case. Upon increasing the degree of polydispersity the fluid-fluid binodal "crosses" the fluid-solid coexistence line and one obtains a stable critical point with associated liquid and gas phases and a triple point. This effect seems to be robust against various ways to incorporate polydispersity, which can be rationalized by the fact that particles of a polydisperse depletion agent can fill the available free volume in a system better than a monodisperse species.

Our main result is the study of mixtures of colloids and 

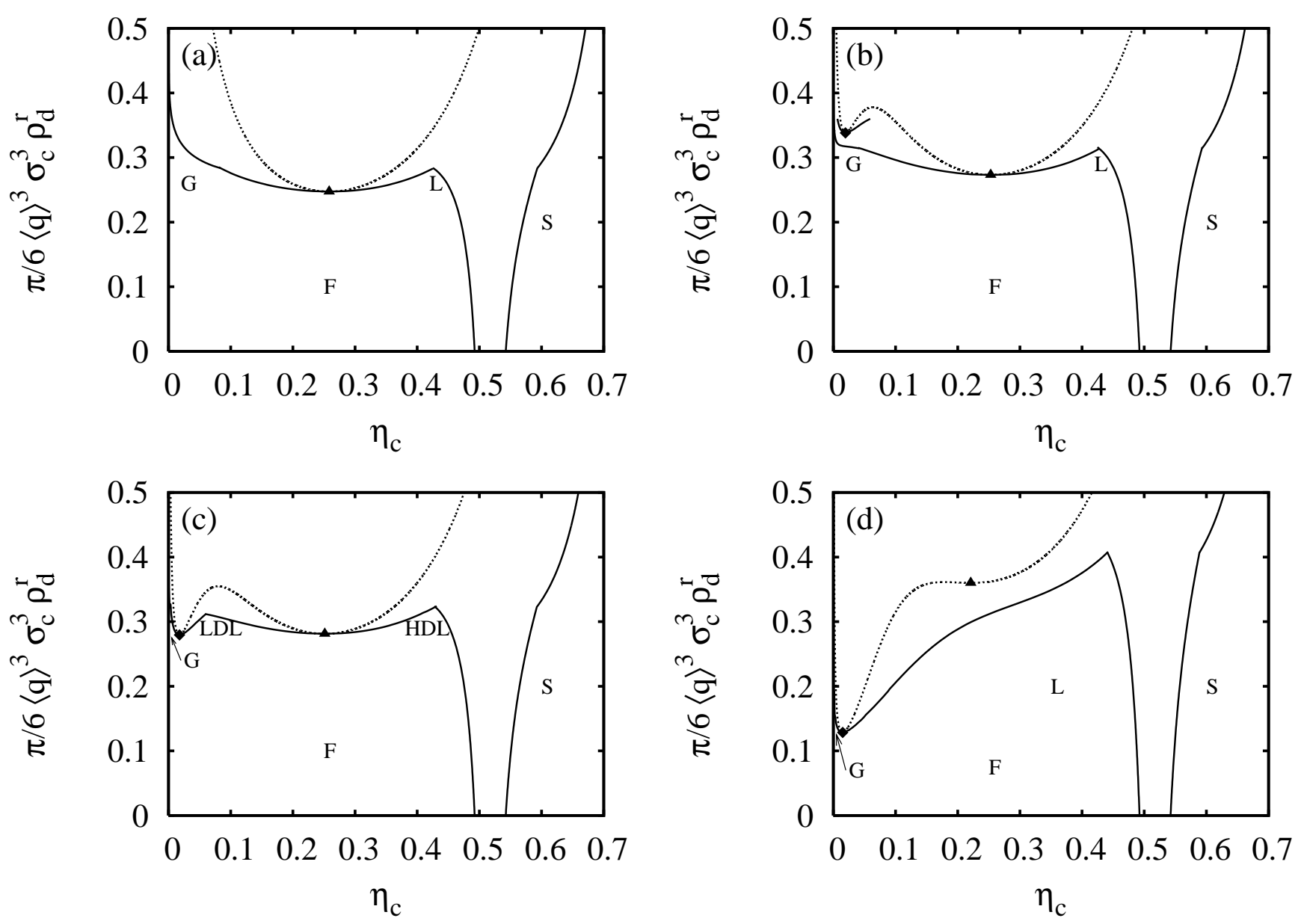

FIG. 4: Phase diagrams for a mixture of colloids and a bimodal distribution of polydisperse (spherical) polymer. For $x$ very close to 1 we recover the phase diagram for the monomodal distribution, see plot (a) for $x=0.9999$. The fluid-fluid coexistence (full line) is stabilized by polydispersity. Upon decreasing $x$, i.e. by adding larger polymer, the spinodal (dotted line) exhibits a second minimum for $x=0.995$ in Fig. (b) and we observe a second critical point, which is metastable (diamond). For $x=0.9935$ we observe two stable critical points. Beside a low density (in colloids) gas (G) there exists a low density (LDL) and a high density (HDL) liquid phase - see Fig. (c). For a value of $x=0.98$, shown in (d), the critical point at high values of $\eta_{c}$ (triangle) is metastable.

two species of polydisperse (spherical) polymer coils. The polymer were described by a bimodal distribution consisting of two polydisperse distributions. For the averaged size ratios of the polymer components chosen here, i.e. $\bar{q}=0.25$ and $\bar{Q}=2.0$, we find in distinct parts of the phase diagram fluid-fluid phase separations in the two limiting cases $x=1$, when only the smaller polymer species is present, and $x=0$, when only the larger polymer species is present. For $x=0$ the corresponding fluid-fluid phase separation is stable independent of the degree of polydispersity. In the second limiting case, $x=1$, the fluid-fluid phase separation has to be stabilized by a sufficient degree of polydispersity. We choose $z_{\bar{q}}=z_{\bar{Q}}=5$ and find for a range of the mixing parameter $x$ two stable liquid phases: beside the gas phase, which is diluted in the density of colloids, we find a low density and a high density liquid phase. The fluid-fluid coexistence region is $\mathrm{w}$-shaped in this case and the phase diagram exhibits two stable critical points and two triple points.

For systems for which the inter-particle interactions contains two length scales, such as short-range repulsion and long-range attraction, Hemmer and Stell14,15 found that two stable critical points may occur. More directly related to our situation is a system of star polymers 16 in which similar features in the phase diagrams are reported, as the effective interaction potential is comparable to those of the model of Stell and Hemmer. For other model fluids similar effects were observed $\underline{38,39,40}$ We point out that our findings are not restricted to a polydisperse depletion agent. We find similar results for a ternary mixture in which we consider one colloid species and two distinct monodisperse species of depletion agent.

While often size or shape polydispersity is considered an undesirable side-effect, originating from imperfections of particle synthesis, it is possible to make use 
of this property by stabilizing fluid-fluid phase separations. Moreover, the degree of polydispersity allows one to adjust the phase diagram. From this point of view one might speculate about fine tuning the phase behavior of colloidal mixtures by specifying the polydisperse distribution of the depletion agent.

\section{APPENDIX A: EXPLICIT EXPRESSIONS FOR $\alpha$ eff}

Explicit expressions for the effective free-volume fraction in the case of infinitely thin needles for the Schulz, Gaussian and Hat-like distributions, Eqs. (16)-(18), read

$$
\begin{aligned}
\alpha_{\mathrm{eff}, S}\left(\eta_{c} ; \bar{q}, z\right)= & \frac{2^{z} z^{z}}{\left(\bar{q} \kappa R_{c}+2 z\right)^{z} e^{\bar{\kappa}}}, \\
\alpha_{\mathrm{eff}, G}\left(\eta_{c} ; \bar{q}, z\right)= & \exp \left(-\bar{\kappa}-\bar{q} \kappa R_{c} / 2+\frac{\kappa^{2} R_{c}^{2}}{16 z^{2}}\right) \\
& \times \frac{1}{\operatorname{erf}(z \bar{q})+1}\left(1-\operatorname{erf}\left(\frac{-z^{2} \bar{q}+\kappa R_{c} / 4}{z}\right)\right), \\
\alpha_{\mathrm{eff}, G}^{\prime}\left(\eta_{c} ; \bar{q}, z\right)= & \exp \left(-\bar{\kappa}-\bar{q} \kappa R_{c} / 2+\frac{\kappa^{2} R_{c}^{2}}{16 z^{2}}\right), \\
\alpha_{\mathrm{eff}, H}\left(\eta_{c} ; \bar{q}, z\right)= & \frac{2 z}{\kappa R_{c}} \exp \left(-\bar{\kappa}-\bar{q} \kappa R_{c} / 2\right) \sinh \left(\frac{\kappa R_{c}}{2 z}\right) .
\end{aligned}
$$

Since only $\kappa$ and $\bar{\kappa}$ enter these expressions for a onedimensional depletion agent, there is no difference between an approach based on the PY or the BMCSL equa- tion of state. In terms of the colloid packing fraction $\eta_{c}$, average asymmetry ratio $\bar{q}$ and the polydispersity parameter $z$ we obtain

$$
\begin{aligned}
\alpha_{\mathrm{eff}, S}\left(\eta_{c} ; \bar{q}, z\right)= & (2 z)^{z}\left(1-\eta_{c}\right)\left(3 \frac{\bar{q} \eta_{c}}{1-\eta_{c}}+2 z\right)^{-z}, \\
\alpha_{\mathrm{eff}, G}\left(\eta_{c} ; \bar{q}, z\right)= & \left(1-\eta_{c}\right) \exp \left(-\frac{3 \bar{q} \eta_{c}}{2\left(1-\eta_{c}\right)}+\frac{9 \eta_{c}^{2}}{16 z^{2}\left(1-\eta_{c}\right)^{2}}\right) \\
& \times \frac{1}{\operatorname{erf}(z \bar{q})+1}\left(1-\operatorname{erf}\left(-z \bar{q}+\frac{3 \eta_{c}}{4 z\left(1-\eta_{c}\right)}\right)\right), \\
\alpha_{\mathrm{eff}, G}^{\prime}\left(\eta_{c} ; \bar{q}, z\right)= & \left(1-\eta_{c}\right) \exp \left(-\frac{3 \bar{q} \eta_{c}}{2\left(1-\eta_{c}\right)}+\frac{9 \eta_{c}^{2}}{16 z^{2}\left(1-\eta_{c}\right)^{2}}\right), \\
\alpha_{\mathrm{eff}, H}\left(\eta_{c} ; \bar{q}, z\right)= & \frac{2 z\left(1-\eta_{c}\right)^{2}}{3 \eta_{c}} \exp \left(\frac{-3 \bar{q} \eta_{c}}{2\left(1-\eta_{c}\right)}\right) \sinh \left(\frac{3 \eta_{c}}{2 z\left(1-\eta_{c}\right)}\right) .
\end{aligned}
$$

In the case of infinitely thin disk- or hexagon-shaped platelets the expressions for the effective free-volume

$$
\begin{aligned}
& \alpha_{\mathrm{eff}, S}\left(\eta_{c} ; \bar{q}, z\right)=\left(\frac{z}{\bar{q}}\right)^{z}\left(-2 \chi_{2}\right)^{-z / 2} \exp \left(-\frac{\chi_{1}^{2}}{8 \chi_{2}}+\chi_{0}\right) D_{-z}\left(\frac{-\chi_{1}}{\sqrt{-2 \chi_{2}}}\right) \\
& \alpha_{\mathrm{eff}, G}\left(\eta_{c} ; \bar{q}, z\right)=\exp \left(\frac{z^{2} \bar{q}^{2} \chi_{2}+\chi_{0} z^{2}-\chi_{0} \chi_{2}+z^{2} \bar{q} \chi_{1}+\chi_{1}^{2} / 4}{z^{2}-\chi_{2}}\right)
\end{aligned}
$$




$$
\begin{gathered}
\times\left(\operatorname{erf}\left(\frac{z^{2} \bar{q}+\chi_{1} / 2}{\sqrt{z^{2}-\chi_{2}}}\right)+1\right) \frac{z}{2[\operatorname{erf}(z \bar{q})+1] \sqrt{z^{2}-\chi_{2}}}, \\
\alpha_{\mathrm{eff}, G}^{\prime}\left(\eta_{c} ; \bar{q}, z\right)=\exp \left(\frac{z^{2} \bar{q}^{2} \chi_{2}+\chi_{0} z^{2}-\chi_{0} \chi_{2}+z^{2} \bar{q} \chi_{1}+\chi_{1}^{2} / 4}{z^{2}-\chi_{2}}\right) \frac{z}{\sqrt{z^{2}-\chi_{2}}} \\
\alpha_{\mathrm{eff}, H}\left(\eta_{c} ; \bar{q}, z\right)= \\
\frac{z}{4} \sqrt{\frac{\pi}{-\chi_{2}}} \exp \left(\chi_{0}-\frac{\chi_{1}^{2}}{4 \chi_{2}}\right) \sum_{n=1}^{2}(-1)^{n} \operatorname{erf}\left(\frac{2 \chi_{2}\left(\bar{q}-(-1)^{n}\right)+\chi_{1} z}{2 z \sqrt{-\chi_{2}}}\right),
\end{gathered}
$$

where $\chi_{2}=-\beta \gamma a_{d} / q^{2}, \chi_{1}=-\beta \kappa c_{d} / q$ and $\chi_{0}=-\beta \bar{\kappa} X_{d}$. The geometrical measures $a_{d}, c_{d}$ and $X_{d}$ are given in
Tab. I. $D_{-z}$ with $z>0$ are parabolic cylinder functions.
1 S. Asakura and F. Oosawa, J. Chem. Phys. 22, 1255 (1954).

2 S. Asakura and F. Oosawa, J. Pol. Sci. 33, 183 (1958).

3 A. Vrij, Pure Appl. Chem. 48, 471 (1976).

4 S.M. Oversteegen and H.N.W. Lekkerkerker, J. Chem. Phys. 120, 2470 (2004).

${ }^{5}$ F.L. Calderon, J. Bibette, and J. Biais, Europhys. Lett. 23, 653 (1993).

6 P.N. Pusey, W.C.K. Poon, S.M. Ilett, and P. Bartlett, J. Phys.: Condens. Matter 6, A29 (1994).

7 D.J. Fairhurst and R.M.L. Evans, Colloid Polym. Sci. 282, 766 (2004).

8 S. Martin, G. Boyant, and W. van Megen, Phys. Rev. E 67, 061405 (2003).

9 J.J. Salacuse and G. Stell, J. Chem. Phys. 77, 3714 (1982).

10 P. Paricaud, S. Varga, P.T. Cummings, and G. Jackson, Chem. Phys. Lett. 398, 489 (2004).

11 R.M.L. Evans, J. Chem. Phys. 114, 1915 (2001).

12 P.B. Warren, Europhys. Lett. 46, 295 (1999).

13 Y. Rosenfeld, Phys. Rev. Lett. 63, 980 (1989).

14 P.C. Hemmer and G. Stell, Phys. Rev. Lett. 24, 1284 (1970).

15 G. Stell and P.C. Hemmer, J. Chem. Phys. 56, 4274 (1972).

16 F. Lo Verso, M. Tau, and L. Reatto, J. Phys.: Condens. Matter 15, 1505 (2003).

17 S.M. Oversteegen and R. Roth, J. Chem. Phys. 122, 214502 (2005).

18 M. Schmidt, H. Löwen, J.M. Brader, and R. Evans, Phys. Rev. Lett. 85, 1934 (2000).

19 M. Dijkstra, J.M. Brader, and R. Evans, J. Phys.: Condens. Matter 11, 10079 (1999).

${ }^{20}$ H.N.W. Lekkerkerker, W.C.K. Poon, P.N. Pusey, A. Stroobants, and P.B. Warren, Europhys. Lett. 20, 559 (1992).

21 D. Frenkel and A.J.C. Ladd, J. Chem. Phys. 81, 3188
(1984).

${ }^{22}$ W.W. Wood, J. Chem. Phys. 20, 1334 (1952).

23 Y. Rosenfeld, Phys. Rev. E 50, R3318, (1994).

24 Y. Rosenfeld, Mol. Phys. 86, 637 (1995).

25 P.-M. König, R. Roth, and K.R. Mecke, Phys. Rev. Lett. 93, 160601 (2004).

${ }^{26}$ H. Hansen-Goos and R. Roth, J. Chem. Phys. 124154506 (2006).

27 R. Roth, R. Evans, A. Lang, and G. Kahl, J. Phys.: Condens. Matter 14, 12063 (2002).

28 Y.X. Yu and J. Wu, J. Chem. Phys. 117, 10156 (2002).

29 T. Boublík, J. Chem. Phys. 53, 471 (1970).

30 G.A. Mansoori, N.F. Carnahan, K.E. Starling, and T.W. Leland, J. Chem. Phys. 54, 1523 (1971).

31 H. Hansen-Goos and R. Roth, J. Phys.: Condens. Matter 18, 8413 (2006).

32 P.B. Warren, Langmuir 13, 4588 (1997).

33 E.J. Meijer and D. Frenkel, J. Chem. Phys. 100, 6873 (1994).

34 R.P. Sear and D. Frenkel, Phys. Rev. E 55, 1677 (1997).

35 M. Fasolo and P. Sollich, J. Phys.: Condens. Matter 17, 797 (2005).

36 D. Goulding and J.P. Hansen, Mol. Phys. 99, 865 (2001).

37 R. Roth, R. Evans, and A.A. Louis, Phys. Rev. E 64, 051202 (2001).

38 G. Franzese, G. Malescio, A. Skibinsky, S.V. Buldyrev, and H.E. Stanley, Nature 409, 692 (2001).

39 S.V. Buldyrev, G. Franzese, N. Giovambattista, G. Malescio, M.R. Sadr-Lahijany, A. Scala, A. Skibinsky, and H.E. Stanley, Physica A 304, 23 (2002).

40 G. Malescio, G. Franzese, G. Pellicane, A. Skibinsky, S.V. Buldyrev, and H.E. Stanley, J. Phys.: Condens. Matter 14, 2193 (2002). 